\title{
Cross-cultural adaptation of the orthognathic quality of life questionnaire (OQLQ) in a Brazilian sample of patients with dentofacial deformities
}

\author{
Marcelo-Carlos Bortoluzzi ${ }^{1}$, Rafael Manfro ${ }^{2}$, Iathanabi-Cathussa Soares ${ }^{3}$, Andréia-Antoniuk Presta ${ }^{4}$
}

\author{
${ }^{1} \mathrm{PhD}$. Stomatology and Maxillofacial Surgery Professor at Santa Terezinha University Hospital and School of Dentistry, Oeste \\ de Santa Catarina University (UNOESC) \\ ${ }^{2}$ PhD Student. Maxillofacial Surgery Professor at Santa Terezinha University Hospital and School of Dentistry, Oeste de Santa \\ Catarina University (UNOESC) \\ ${ }^{3}$ DDS. Assistant research \\ ${ }^{4}$ Ph.D. Public Health Professor at Oeste de Santa Catarina University (UNOESC)
}

Correspondence:

Universidade do Oeste de Santa Catarina (UNOESC),

Faculdade de Odontologia,

Av. Getúlio Vargas, 2125,

Bairro Flor da Serra-Joaçaba,

Santa Catarina/ Brazil. CEP (ZIP) 89600-000

mbortoluzzi@gmail.com

Bortoluzzi MC, Manfro R, Soares IC, Presta AA. Cross-cultural adaptation of the orthognathic quality of life questionnaire (OQLQ) in a Brazilian sample of patients with dentofacial deformities. Med Oral Patol Oral Cir Bucal. 2011 Aug 1;16 (5):e694-9.

http://www.medicinaoral.com/medoralfree01/v16i5/medoralv16i5p694.pdf

Received: 05/03/2010

Accepted: $31 / 05 / 2010$

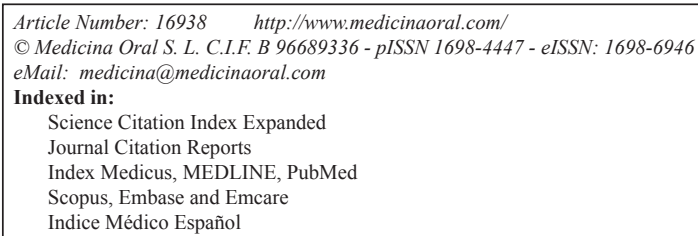

\begin{abstract}
Objectives: The aim of this study is to translate into Portuguese-Brazilian language and adapt cross-culturally to the Brazilian population the Orthognathic Quality of Life Questionnaire (OQLQ).

Study Design: The cross-cultural adaptation process followed six stages which are; (I) initial translation, (II) synthesis of the translation, (III) back translation, (IV) expert committee and (V) test of the prefinal version. For validation process, the OQLQ results were compared with Oral Health Impact Profile Questionnaire (OHIP-49), with the generic SF-36 Quality of Life Questionnaire and a visual analogue scale. A convenience sample of 25 patients was selected in two Southern Brazilian states of Santa Catarina and Rio Grande do Sul.

Results: Internal consistency analysis of the OQLQ-Brazilian showed good correlation for items or domains and the test-retest reliability also presented excellent intra-class correlation coefficients. OQLQ-Brazilian exhibited a weak and negative correlation with SF-36, and good correlation with OHIP-49.

Conclusion: The OQLQ-Brazilian preserved and reached equivalence with its original source and the findings also corroborate that there is good evidence for the construct validity. The demonstration of its reproducibility, reliability and validity makes this instrument an additional useful parameter for evaluation of the impact of dentofacial deformity over the quality of life for Brazilians.
\end{abstract}

Key words: Dentofacial deformity, quality of life, orthognathic quality of life questionnaire, oral health impact profile questionnaire, generic SF-36 questionnaire. 


\section{Introduction}

The World Health Organization (WHO) defined quality of life (QOL) as "an individual's perception of their position in life in the context of the culture and value systems in which they live and in relation to their goals, expectations, standards and concerns" (1). This concept broadened the focus in the measurement of health beyond traditional health indicators such as mortality and morbidity, to include measures of the impact of disease and impairment on daily activities and behavior (2).

Self reported measures of health can serve as a method to build up an understanding of clinical relevance and importance, and these meanings, to be compared between different cultures and nations, necessitate not only to be well translated linguistically but also be culturally adapted to maintain the content validity of the instrument and reach equivalence between the original source and target versions of the questionnaire $(3,4)$. According to Cunningham et al. (5) an increased interest in health-related quality of life is happening due to the possibility to widen the range of outcome measures used in clinical researches, to audit the performance of groups or individuals and to monitor health levels in the community.

Dentofacial deformity (DDF) has been defined as a deformity that primarily affects the jaws and dentition although it also affects the proportions of the mid and lower face and, in the context of QOL, DDF can produce adverse effects over self-esteem and self-confidence and it may evoke undesirable social response or restrict one's lifestyle $(6,7)$. The aim of this study is to translate into Portuguese-Brazilian language and adapt crossculturally to the Brazilian population the Orthognathic Quality of Life Questionnaire (5), in order to provide a measurable condition-specific oral health-related quality of life instrument for patients carrying DDF. This questionnaire may also serve to evaluate the impact or outcome of orthognathic treatment over the QOL in these patients.

\section{Material and Methods}

This study is a cross-cultural adaptation into Portuguese-Brazilian language of the Orthognathic Quality of Life Questionnaire (OQLQ) which was developed (5) and validated (8) by Cunningham et al. in the years 2000 (5) and 2002 (8), respectively. This study was submitted to the Ethical Committee for Human Research and all participants provided a signed informed consent. In this research, the patients included were about to start treatment or in the early stages of pre-surgical orthodontic treatment and with diagnosis of mandibular prognathism, mandibular retrognathism, anterior open bite, laterognathism, vertical maxillary excess or a combination of these health problems. Previous orthognathic surgery, trauma sequel and edentate patients were excluding criteria.
The cross-cultural adaptation process followed the guidelines proposed by Beaton et al. (4), which comprised six stages: Stage I, initial translation; Stage II, synthesis of the translation; Stage III, back translation; Stage IV, expert committee; and Stage V, test of the prefinal version. The section (Stage VI) was a suggested appraisal process whereby an advisory committee or the developers reviewed the process and determined whether this is an acceptable translation. To the validation process, the results of the OQLQ were compared with Oral Health Impact Profile Questionnaire (OHIP$49)$ and with the generic SF-36 QOL questionnaire.

The OQLQ consists of 22 items on a 4-point scale rating and coded as follows: from 1 or "it bothers you a little" to 4 or "it bothers you a lot", being 2 and 3 in between; and NA or "the statement does not apply to you or does not bother you." The 22 items are divided into concerns or domains regarding as social aspects of deformity (first component), facial aesthetics (second component), oral function (third component) and awareness of facial deformity (fourth component). The scoring of the OQLQ is performed by addition of individual items within the domains. A total OQLQ score can range from 0 to 88 , with domains counting specifically (social aspects domain, $0-32$; dento-facial aesthetics domain, $0-20$; function domain, $0-20$; and awareness of dentofacial aesthetics, $0-16)$. A higher score indicates poorer QOL and a lower score better QOL $(5,8)$.

The OHIP questionnaire is a 49 -question instrument developed by Slade and Spencer (9) and was conceived to measure how different oral conditions affect quality of life in an overall sense. It is organized in seven conceptual impact dimensions: "functional limitations", "physical pain", "psychological discomfort", "physical disability", "psychological disability", "social disability" and "handicap". The OHIP-49 domains range from $0-40$ and the values are obtained through a weighted average of the questions which compose each dimension. Higher scores indicate a worse oral health-related quality of life state. The Portuguese version of the OHIP-49, was adapted to the Brazilian culture by Pires et al. (10). The Brazilian version of the generic SF-36 consists of 36 statements divided into 8 domains: physical functioning, physical role, bodily pain, general health, vitality, social functioning, emotional role and mental health. For each subscale, raw data are transformed and summed on a $0-100$ scale with a higher score indicating better health state $(10,11)$.

A convenience sample of 25 patients was selected in two Southern Brazilian states of Santa Catarina and Rio Grande do Sul. The patients were given 3 questionnaires (OQLQ, OHIP and SF-36) and a visual analogue scale (VAS) ranging from 0 to 100 (with higher scores indicating better health state), this last bringing information and concepts of generic QOL. Instructions about how to 
answer the questionnaires were given to the patients by a Maxillofacial Surgeon enrolled in the research, so any doubt over the questions could be solved and annotated to possibly review the OQLQ/Brazilian version. When all questionnaires and VAS where filled up and collected, patients answered a second OQLQ (OQLQ-T2). Data were analyzed through statistical software STATA 8.0. The analysis involved firstly an evaluation of the translated instrument concerning its internal consistency based on the correlation among questions and its domains and, the replicability (test-retest) of the questionnaire. Secondly validation and cross-cultural adaptation of the instrument was analyzed through correlation between the OQLQ-Brazilian and OHIP-49, SF-36 and a visual analogue scale.

\section{Results}

Female patients were 64\% (16 patients) of the sample with age ranging from 11 to 52 years (mean 25.4, SD
12.1). The internal consistency analysis to check the reliability and structure of the instrument of item-domains of the OQLQ-Brazilian can be viewed in the (Table 1). All domains of the Brazilian translated instrument showed good correlation for items and domains. A low item-total correlation would indicate that the item was unrelated to the items in the remainder of the scale. In a very similar way to the original OQLQ questionnaire the question 1 in the second and question 5 in the third components gave values below 0.5. In OQLQ-Brazilian the question 8 of the fourth component also gave values below 0.5 , however borderline $(0.49)$. The test-retest reliability of the OQLQ-Brazilian (OQLQ versus OQLQT2) showed excellent intra-class correlation coefficients, equal or superior to 0.94 .

The OQLQ scores values showed that the facial aesthetics was the most important domain affecting the patient QOL in subjects carrying dentofacial deformity, followed by oral function and social aspects of defor-

Table 1. Internal consistency analysis for the domains of the Orthognathic Quality of Life Questionnaire/Brazilian Version $(\mathrm{n}=25)$.

\begin{tabular}{|c|c|c|c|}
\hline Component/item & $\begin{array}{l}\text { Item-total } \\
\text { correlation }\end{array}$ & $\begin{array}{c}\text { Alpha coefficient } \\
\text { for each } \\
\text { component }\end{array}$ & $\begin{array}{l}\text { Intra-class } \\
\text { correlation } \\
\text { coefficient }\end{array}$ \\
\hline \multicolumn{4}{|l|}{ Component 1 - social aspects of deformity } \\
\hline 15. Cover mouth when meeting people & 0.66 & & \\
\hline 16. Worry about meeting people & 0.77 & & \\
\hline 17. Worry people will make hurtful comments & 0.79 & & \\
\hline 18. Lack confidence socially & 0.73 & 0.89 & $0.89(\mathrm{p}<0.001)$ \\
\hline 19. Do not like smiling & 0.58 & & \\
\hline 20. Get depressed about appearance & 0.60 & & \\
\hline 21. Sometimes think people are staring & 0.86 & & \\
\hline 22. Comments about appearance upset me & 0.58 & & \\
\hline \multicolumn{4}{|l|}{ Component 2 - facial aesthetics } \\
\hline 1. Self-conscious about appearance of my teeth & 0.23 & & \\
\hline 7. Don't like seeing side view of face (profile) & 0.61 & & \\
\hline 10. Dislike having photograph taken & 0.75 & 0.78 & $0.78(\mathrm{p}<0.001)$ \\
\hline 11. Dislike being seen on video & 0.65 & & \\
\hline 14. Self-conscious about appearance & 0.58 & & \\
\hline \multicolumn{4}{|l|}{ Component 3 -oral function } \\
\hline 2. Problems biting & 0.82 & & \\
\hline 3. Problems chewing & 0.70 & & \\
\hline 4. Avoid eating some foods & 0.60 & 0.78 & $0.78(\mathrm{p}<0.001)$ \\
\hline 5. Don't like eating in public & 0.10 & & \\
\hline 6. Pains in face/jaw & 0.63 & & \\
\hline \multicolumn{4}{|l|}{ Component 4 - awareness of facial deformity } \\
\hline 8. Spend time studying face & 0.49 & & \\
\hline 9. Spend time studying teeth & 0.72 & 0.82 & $0.82(\mathrm{p}<0.001)$ \\
\hline 12. Stare at people's teeth & 0.73 & & \\
\hline 13. Stare at people's faces & 0.63 & & \\
\hline
\end{tabular}


Table 2. Spearman's rho correlation matrix between Orthognathic Quality of Life Questionnaire/ Brazilian Version and Generic SF-36 Questionnaire, Oral Health Impact Profile (OHIP-49) and a Visual Analogue Scale (n=25).

\begin{tabular}{|c|c|c|c|c|}
\hline & \multicolumn{4}{|c|}{$\begin{array}{c}\text { OQLQ Domains } \\
\text { Spearman's rho Correlation (1) }\end{array}$} \\
\hline & $\begin{array}{c}\text { Social } \\
\text { aspects of } \\
\text { deformity } \\
\end{array}$ & $\begin{array}{c}\text { Facial } \\
\text { aesthetics }\end{array}$ & $\begin{array}{c}\text { Oral } \\
\text { function }\end{array}$ & $\begin{array}{c}\text { Awareness of } \\
\text { facial } \\
\text { deformity }\end{array}$ \\
\hline \multicolumn{5}{|l|}{ SF-36 (2) } \\
\hline Physical functioning & 0.17 & -0.09 & -0.31 & -0.07 \\
\hline Role limitations due to physical health & 0.15 & -0.18 & $-0.55 * *$ & -0.38 \\
\hline Role limitations due to emotional problems & 0.39 & -0.17 & -0.22 & -0.2 \\
\hline Energy/ fatigue & 0.74 & -0.26 & -.0 .33 & -0.31 \\
\hline Emotional well being & -0.16 & -0.23 & $-0.57 * *$ & -0.27 \\
\hline Social functioning & -0.14 & -0.27 & $-0.47 * *$ & -0.26 \\
\hline Pain & 0.15 & $-0.4^{*}$ & $-0.41^{*}$ & $-0.39 *$ \\
\hline General health & 0.05 & -0.04 & -0.14 & 0.22 \\
\hline \multicolumn{5}{|l|}{ OHIP-49 (3) } \\
\hline Functional limitation & 0.11 & $0.35^{*}$ & $0.91 * *$ & 0.2 \\
\hline Physical pain & 0.15 & 0.13 & $0.84 * *$ & 0.01 \\
\hline Psychological discomfort & 0.12 & $0.48 * *$ & $0.81 * *$ & $0.48 * *$ \\
\hline Physical disability & 0.3 & 0.25 & $0.89 * *$ & 0.2 \\
\hline Psychological disability & 0.1 & 0.32 & $0.7 * *$ & $0.44 *$ \\
\hline Social disability & 0.33 & 0.06 & $0.63 * *$ & 0.31 \\
\hline Handicap & 0.00 & $0.42 *$ & $0.82 * *$ & 0.33 \\
\hline \multicolumn{5}{|l|}{ Visual Analogue Scale (4) } \\
\hline VAS & .004 & .45 & .69 & .47 \\
\hline
\end{tabular}

*Correlation is significant at the 0.05 level (1-tailed).

**Correlation is significant at the 0.01 level (1-tailed).

(1) OQLQ: higher score indicates poorer quality of life.

(2) OHIP-49: higher score indicates poorer quality of life.

(3) SF36: higher score indicates better quality of life.

(4) VAS: higher score indicates better quality of life.

mity. The OHIP domains of psychological discomfort, functional limitation and physical pain showed to have higher impact over QOL in patients with dentofacial deformity. Poorer QOL in this group of patients, evaluated through SF-36 generic form, was observed for domains energy/ fatigue, general health, role limitations due to physical health and emotional well-being.

The correlation matrix between the domains of the OQLQ and SF-36, OHIP and a visual analogue scale can be viewed in (Table 2).

\section{Discussion}

Dentofacial deformity has shown to influence several aspects of patients' psychologic, social and physical status, which can profoundly impair individuals' QOL and affect important life aspects of function, appearance and interpersonal relationships $(5,7,8,12)$ and besides this, QOL in patients with DDF in Brazilian population is almost unknown (11,13). QOL for a Brazilian sample of 29 patients carrying DDF and submitted to orthognathic surgery were evaluated through SF-36 generic form by Nicodemo et al. (11) and the authors observed that of the eight domains of the questionnaire, four domains showed improvement after the surgery (vitality, emotional, physical and social aspects). In the present manuscript, evaluating through the same questionnaire, it was observed that vitality is the most important aspect affecting quality of life in patients carrying dentofacial deformity.

To investigate QOL in patients with DDF, various approaches have been undertaken, with no consensus on a standard method of assessment and the limited responsiveness of generic measurements to evaluate oral conditions or diseases highlights the importance of developing oral condition-specific QOL measurements $(7,11,12,14,15)$. This lack of consensus and information 
about QOL in patients with DDF in Brazilian population, also suggests the necessity of having a comparable and condition-specific QOL instrument.

Al-Ahmad et al. (7) evaluated three groups of patients with DDF (presurgery, postsurgery and declined surgery) and a control group with the SF-36 generic form and observed that this instrument was weak to discriminate between the 4 groups, while the OQLQ was highly discriminative. As found by Al-Ahmad et al. (7), this present study found a weak correlation between the domains of the OQLQ and the generic SF-36 questionnaire, showing mainly that the "oral function" domain of the OQLQ was correlated with only 4 domains of the SF-36, and when the total mean values of both instruments were analyzed a weak negative correlation was found between both questionnaires, proving that the SF36 could be insensitive for specific clinical oral conditions, such as in patients with dentofacial deformities. Interestingly, when the original OQLQ was compared with the SF-36 by Cunningham et al. (8), the "oral function" domain showed only one significant correlation with all SF-36 scales and those authors also confirmed that there exist poor levels of correlation between the OQLQ domains and the SF-36 scales.

Comparisons between OQLQ-Brazilian and OHIP showed strong correlation between all domains of the OHIP and OQLQ "oral function" domain and other three associations were found between OQLQ "facial aesthetics" and OHIP (functional limitation, psychological discomfort and handicap), and two between OQLQ "awareness of facial deformity" and OHIP (psychological discomfort and psychological disability). Therefore, the present study also indicates a good validity of the OQLQ-Brazilian due to its ability to detect the impact of the QOL in patients with DDF, which were comparable to the OHIP results. However, when compared with SF-36 data, these results indicate that when more condition-specific is the QOL questionnaire, more easily is detected the impact over QOL in these patients. Similar data were found by Choi et al. (16), who evaluated 32 patients with DDF before and after orthodontic-surgical treatment with the generic form of the SF-36, a short form of the OHIP-14 and OQLQ, and they found just one domain of the SF-36 showing changes, while five of the seven OHIP domains and two of four domains of the OQLQ detected changes. In the same way, Lee et al. (15), in a case-control study, found no differences between case and control patients with and without DDF, respectively, using SF-36 generic form. Nevertheless, they found significant differences in overall OHIP-14 scores and overall OQLQ scores between case and control groups.

The VAS scores obtained in this sample showed no correlations between all questionnaires, which means that a single item measure is less reliable and don't carry the ability to capture all patients' concerns. This finding is also in accordance with the results of Cunningham et al. (8). On the other hand, Modig et al. (12) have used a visual analogue scale (VAS) to measure chewing problems, digestive problems, headache, snoring problems, speech problems, aesthetic problems and bullying in patients with DDF and submitted to orthognathic surgery, and found out that $72 \%$ of those subjects thought that their condition after the operation was much better than before. Those authors also used the OHIP-49 questionnaire, however that manuscript didn't bring any detailed results of the questionnaire use.

Different systems of values and culture could explain a slightly lower mean OQLQ value domains (better QOL) found in the Brazilian sample when compared to British (8), Chinese (15) and Jordanian patients with DDF (7). The Brazilian version of the OQLQ showed to be a simple and easy language questionnaire that could be understood by individuals from any socio-cultural level and even to children with age as low as eleven years old. The results indicated that the OQLQ-Brazilian preserved and reached equivalence with its original source and the findings also corroborate that there is good evidence for the construct validity. The demonstration of its reproducibility, reliability and validity makes this instrument an additional useful parameter for evaluation of the impact of dentofacial deformity over the quality of life for Brazilians.

\section{References}

References with links to Crossref - DOI

1. Study protocol for the World Health Organization project to develop a Quality of Life assessment instrument (WHOQOL). Qual Life Res. 1993;2:153-9.

2. Division of mental health and prevention of substance abuse, World Health Organization. Measuring quality of life [Internet]. World Health Organization; 1997 [cited 2009 Jan 5]. Available from: // www.who.int/mental_health/media/68.pdf. Accessed in 05/01/2009. 3. Beaton DE. Understanding the relevance of measured change through studies of responsiveness. Spine (Phila Pa 1976). 2000;25:3192-9.

4. Beaton DE, Bombardier C, Guillemin F, Ferraz MB. Guidelines for the process of cross-cultural adaptation of self-report measures. Spine (Phila Pa 1976). 2000;25:3186-91.

5. Cunningham SJ, Garratt AM, Hunt NP. Development of a condition-specific quality of life measure for patients with dentofacial deformity: I. Reliability of the instrument. Community Dent Oral Epidemiol. 2000;28:195-201.

6. Cunningham SJ, Hunt NP, Feinmann C. Perceptions of outcome following orthognathic surgery. Br J Oral Maxillofac Surg. 1996;34:210-3.

7. Al-Ahmad HT, Al-Sa'di WS, Al-Omari IK, Al-Bitar ZB. Condition-specific quality of life in Jordanian patients with dentofacial deformities: a comparison of generic and disease-specific measures. Oral Surg Oral Med Oral Pathol Oral Radiol Endod. 2009;107:4955 .

8. Cunningham SJ, Garratt AM, Hunt NP. Development of a condition-specific quality of life measure for patients with dentofacial deformity: II. Validity and responsiveness testing. Community Dent Oral Epidemiol. 2002;30:81-90.

9. Slade GD, Spencer AJ. Development and evaluation of the Oral Health Impact Profile. Community Dent Health. 1994;11:3-11.

10. Pires CP, Ferraz MB, De Abreu MH. Translation into Brazilian 
Portuguese, cultural adaptation and validation of the oral health impact profile (OHIP-49). Braz Oral Res. 2006;20:263-8.

11. Nicodemo D, Pereira MD, Ferreira LM. Effect of orthognathic surgery for class III correction on quality of life as measured by SF36. Int J Oral Maxillofac Surg. 2008;37:131-4.

12. Modig M, Andersson L, Wårdh I. Patients' perception of improvement after orthognathic surgery: pilot study. Br J Oral Maxillofac Surg. 2006;44:24-7.

13. Marques LS, Ramos-Jorge ML, Paiva SM, Pordeus IA. Malocclusion: esthetic impact and quality of life among Brazilian schoolchildren. Am J Orthod Dentofacial Orthop. 2006;129:424-7.

14. Motegi E, Hatch JP, Rugh JD, Yamaguchi H. Health-related quality of life and psychosocial function 5 years after orthognathic surgery. Am J Orthod Dentofacial Orthop. 2003;124:138-43.

15. Lee S, McGrath C, Samman N. Quality of life in patients with dentofacial deformity: a comparison of measurement approaches. Int J Oral Maxillofac Surg. 2007;36:488-92.

16. Choi WS, Lee S, McGrath C, Samman N. Change in quality of life after combined orthodontic-surgical treatment of dentofacial deformities. Oral Surg Oral Med Oral Pathol Oral Radiol Endod. 2010;109:46-51.

\section{Acknowledgments}

The authors wish to thank to Miguel Scheffer, Marcos Tomazi and Silvio Gallon for their help and especially for Dr. Susan J. Cunningham (UCL Eastman Dental Institute) for her consent and support in this research. 